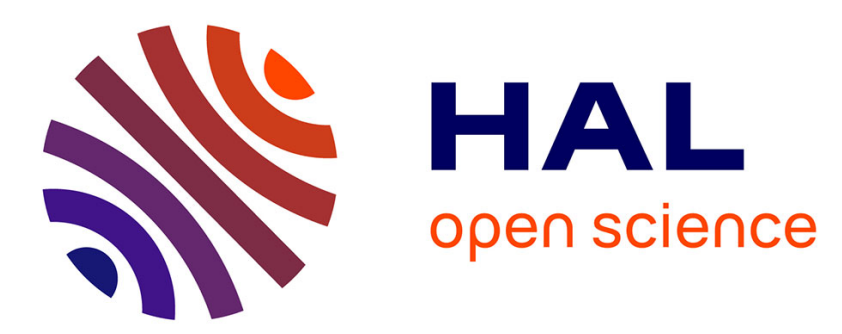

\title{
Angular assessment of the Mojette filtered back projection
}

Peggy Subirats, Myriam Servières, Nicolas Normand, Jeanpierre Guédon

\section{To cite this version:}

Peggy Subirats, Myriam Servières, Nicolas Normand, Jeanpierre Guédon. Angular assessment of the Mojette filtered back projection. Medical Imaging 2004, 2004, San Diego, United States. pp.19511960, 10.1117/12.533483 . hal-01500582

\section{HAL Id: hal-01500582 \\ https://hal.science/hal-01500582}

Submitted on 16 May 2017

HAL is a multi-disciplinary open access archive for the deposit and dissemination of scientific research documents, whether they are published or not. The documents may come from teaching and research institutions in France or abroad, or from public or private research centers.
L'archive ouverte pluridisciplinaire HAL, est destinée au dépôt et à la diffusion de documents scientifiques de niveau recherche, publiés ou non, émanant des établissements d'enseignement et de recherche français ou étrangers, des laboratoires publics ou privés. 


\title{
Angular Assessement of the Mojette Filtered Back Projection
}

\author{
Peggy Subirats, Myriam Servières, Nicolas Normand and Jeanpierre Guédon \\ IRCCyN - UMR CNRS 6597, Image \& Video Communication, \\ École polytechnique de l'Université de Nantes \\ La Chantrerie, BP 50609, 44306 Nantes Cedex 3
}

\begin{abstract}
In this paper, we propose to perform a novel discrete implementation of the filtered back projection algorithm. For this, we use a version of the discrete exact Radon transform called the Mojette transform that has been developed in our team for few years. The initial questioning was centered about the angular distribution needed for the continuous Radon reconstruction. Because of the discrete set of angles used in the FBP algorithm, discrete angles issued from Farey's series were used. Our version of the FBP algorithm is compared with the classical FBP algorithm. The choice of the set of projection angles is discussed in order to produce a good and efficient angular sampling. Finally, the very different behaviors between the classical FBP and our algorithm justify our study.
\end{abstract}

Keywords: tomographic reconstruction, discrete Radon transform, filtered back projection, Mojette transform, angular sampling

\section{INTRODUCTION}

FBP is the most implemented tomographic algorithm. However, this scheme directly derives from a continuous linear decomposition of the inverse Radon transform without a clear and unique way of sampling this process. ${ }^{1-3}$ It is commonly well admitted that a regular angular sampling is mandatory for FBP reconstructions. A regular angular sampling is the best way to divide space in a continuous mode.

The goal of this paper is to explore the relationship between angular sampling and tomographic data (discrete projections). As soon as the original 2D function is represented from a grid and its samples, the previous regular angular sampling becomes (at least) unproven as the correct one. The natural counterpart of discrete projection sampling is the use of Farey's series. ${ }^{4}$ The obtained discrete angles constitute the basis for the Radon discrete version called the Mojette transform.

In this paper, we propose to implement a new discrete filtered back projection algorithm both from an angular and a linear sampling point of view. For this, we use a version of the discrete exact Radon transform called the Mojette transform that has been developed in our team for few years. Our version of the FBP algorithm is compared with the classical FBP algorithm. Finally we discuss the choice of the projection angles set leading to an optimal tradeoff between a low number of projections and the best reconstructed object.

\section{FBP MOJETTE}

In this part, we explain the FBP Mojette method. To begin with, we define the Mojette projection mechanism with some reminds on the discrete Radon transform and some properties of the Mojette transform. Then, we explain the Mojette backprojection algorithm and to finish this part on the FBP Mojette, we define the $k_{0}$ filter.

\subsection{Mojette Projection}

The Mojette transform was derived from the Radon transform, ${ }^{5}$ when restricting the projection angles class and making adequate equivalent sampling onto the projections. Doing so in the projection space is consistent with the conditions of the generalized sampling theorem ${ }^{6,7}$ in Spline spaces.

Email: \{peggy.subirats, myriam.servieres, jean-pierre.guedon, nicolas.normand\}@polytech.univ-nantes.fr 


\subsubsection{Discrete Radon transform}

The Radon transform is given in its continuous mode by:

$$
\operatorname{proj}(t, \theta)=\mathbf{R} f(x, y)=\int_{-\infty}^{+\infty} \int_{-\infty}^{+\infty} f(x, y) \delta(t+x \sin \theta-y \cos \theta) d x d y .
$$

The fonctional projection of $f(x, y)$ onto the space defined by its Riesz basis $\{\varphi(x-k), k \in \mathbb{Z}\}$ leads to the interpolation equation :

$$
f_{\varphi}(x, y)=\sum_{k=-\infty}^{+\infty} \sum_{l=-\infty}^{+\infty} f(k, l) \varphi(x-k) \varphi(y-l)
$$

Using Eq.1 to project the grid used in Eq.2 leads to the major choice of only using discrete angles of the form $\tan \theta=\frac{q}{p}$ where $p$ and $q$ are integers (moreover, a single representant of the angles class is kept by restricting $p$ and $q$ to be prime together and $q$ to be positive). In other words, the Riesz basis is replaced with Dirac distribution $\{\varphi(x-k)=\delta(x-k), k \in \mathbb{Z}\}$. Notice that Mojette definitions can also be obtained by taking other Riesz basis choices. For instance the Spline 0 basis $\varphi(x-k)=\left\{\begin{array}{l}1 \text { if }|x-k|<\frac{1}{2} \\ \frac{1}{2} \text { if }|x-k|=\frac{1}{2} \\ 0 \text { elsewhere }\end{array}\right.$ leads to the Spline 0 Mojette definition. $^{8}$

Effectively, when jointly using such an angle with the projection sampling (where $b$ is called a bin) leads to a regular lattice with keeping all pixels centers onto projection bins. In this case, Eq.1 is reduced to ${ }^{9}$ :

$$
\operatorname{proj}(b, p, q)=M_{\delta} f(k, l)=\sum_{k=-\infty}^{+\infty} \sum_{l=-\infty}^{+\infty} f(k, l) \Delta(b+q k-p l),
$$

where $\Delta(b)=\left\{\begin{array}{lll}0 & \text { if } & b \neq 0 \\ 1 & \text { if } \quad b=0\end{array}\right.$ is the discrete Kronecker symbol. Eq.3 defines the Mojette transform operator (see Fig. 1).

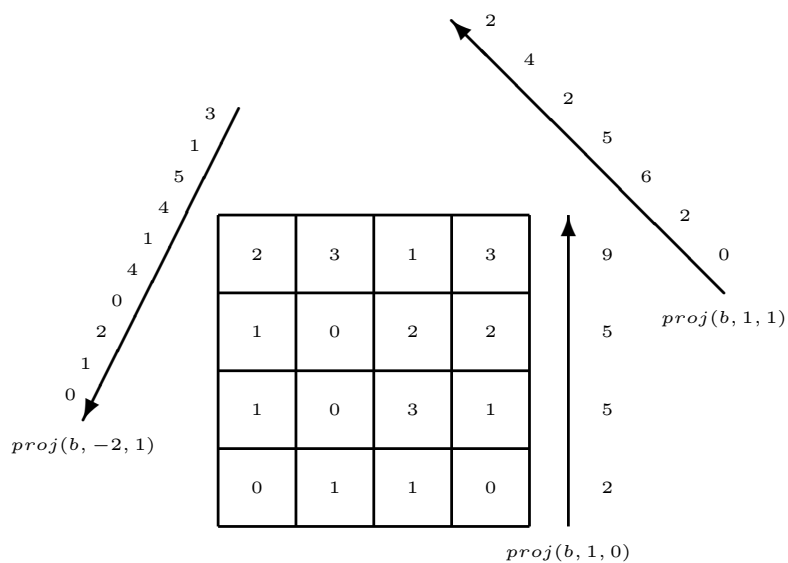

Figure 1. Mojette projections of a $4 \times 4$ image with projections directions $(p, q) \in\{(1,0),(1,1),(-2,1)\}$ 


\subsubsection{Mojette transform properties}

The 2D Mojette transform defined in Eq.3 has some interesting properties. Linearity and shift-invarianceness of the Radon transform are kept because both angular and projection samplings are realized in an adequate manner. Reconstruction can be exactly performed with a finite number of angles when perfect data are involved. ${ }^{10}$

The main characteristic of the Mojette transform lies in the sampling (first presented by Katz ${ }^{11}$ ). Another way of seeing this is to say that the linear system expressed in Eq.3 is exactly invertible because of the sampling. From a rectangular image $Q \times P$, this matrix inversion must be possible to perform an inverse mojette transform, i.e. a minimum number of projections is required. For the rectangular shape, the Katz criterion allows exact reconstruction if:

$$
P \leq \sum_{i=1}^{I}\left|p_{i}\right| \text { or } Q \leq \sum_{i=1}^{I}\left|q_{i}\right|,
$$

where $I$ is the number of projections. In other words, the reconstruction does not depend directly on the number of bins but is first related to the number of projections. These reconstruction conditions have been generalized for any convex shape using a simple morphological tool : a two pixel structuring element that depicts a discrete direction. ${ }^{5}$

The inverse Mojette operator is also defined in a specific way. Instead of a matrix inversion (which is possible but costly), the operator only proceeds by finding at each iteration a discrete corner of the shape under reconstruction which is the only projected pixel onto a bin: the bin value is exactly backprojected and all projections are updated. The algorithm complexity of both the direct and inverse Mojette transform is $O(I N)$ where $N$ is the number of pixels.

\subsection{Mojette Backprojection}

In this paragraph, we define the Mojette Backprojection operator which will be used in the FBP Mojette scheme. For a single angle $(p, q)$ the discrete backprojector is defined by ${ }^{10}$ :

$$
\begin{aligned}
\tilde{f}(k, l) & =M^{*} \operatorname{proj}(b, p, q) \\
& =\sum_{i} \sum_{j} \delta(k-i) \delta(l-j) \sum_{b} \operatorname{proj}(b, p, q) \Delta(b+q i-p j) .
\end{aligned}
$$

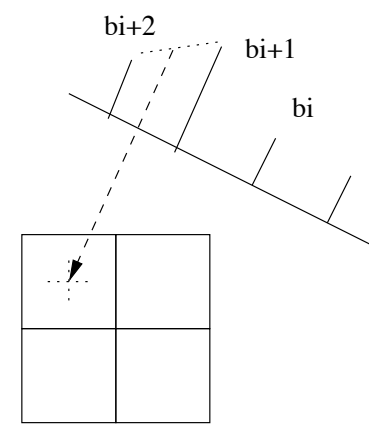

(a)

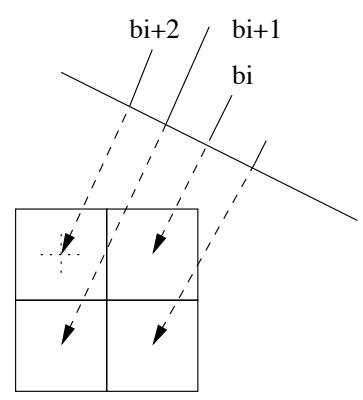

(b)

Figure 2. (a) Interpolation onto the projection in order to backproject the right value at the corresponding center of the pixel, (b) Exact Mojette backprojector. 
The Mojette dual operator $M^{*}$ corresponds to an exact discrete backprojector. As depicted in Fig.2.a, the classical FBP needs to perform an approximation via an interpolator scheme implemented onto the projection to get the backprojected value at the center of the pixels. In the Mojette case (Fig.2.b), no interpolation is required since the sampling onto the projection exactly matches the pixel sampling.

\subsection{FBP Mojette algorithm}

This section describes the $k_{0}$ filter ${ }^{1}$ adapted to the Mojette geometry and then the FBP Mojette algorithm used for the reconstruction is given.

\subsection{1. $k_{0}$ filter}

The continuous exact FBP Spline 0 filter expression in the Fourier domain is given by:

$$
K_{0}(\nu, \theta)=\pi|\nu| \operatorname{sinc}(\nu \cos \theta) \operatorname{sinc}(\nu \sin \theta)
$$

where the apodisation function corresponds to the projection of the filter onto the Spline of order 0 space. The pixel size is $1 \times 1$.

The inverse Fourier transform of Eq. 6 was derived as

$$
k_{0}(t, \theta)=\frac{1}{\pi \sin (2 \theta)} \ln \left|\frac{t^{2}-\left(\frac{1+\sin (2 \theta)}{4}\right)}{t^{2}-\left(\frac{1-\sin (2 \theta)}{4}\right)}\right|
$$

for $t \neq 0$ and $\left.\theta \in] 0, \frac{\pi}{4}\right]$ and $k_{0}(t, 0)=\frac{-1}{\pi} \frac{2}{4 t^{2}-1}$.

Discretizing with Mojette angles $\left(\tan \theta=\frac{q}{p}\right)$ with the projection sampling $t=\frac{b}{\sqrt{p^{2}+q^{2}}}$ leads to:

$$
k_{0}(b, p, q)=\frac{p^{2}+q^{2}}{2 \pi p q} \ln \left|\frac{b^{2}-\left(\frac{p+q}{2}\right)^{2}}{b^{2}-\left(\frac{p-q}{2}\right)^{2}}\right|,
$$

for $b \neq 0$ and $(p, q) \neq(1,0)$ and $k_{0}(b, 1,0)=\frac{-1}{\pi} \frac{2}{4 b^{2}-1}$.

Notice that this filter can not be implemented in a straightforward manner. It exhibits discontinuities at the locations where the projection of the pixel (which is described by a trapezoidal shape) is continuous but not differentiable. However a Dirichlet condition can be applied to obtain a stable version.

\subsubsection{Algorithm principle}

Here, we replace the Mojette inverse operator usually used by a FBP scheme in the following manner.

First, the continuous expression of the filter recalled in Eq.6 is sampled according to the projection angle $(p, q)$ at a rate $h=\frac{\Delta}{\sqrt{p^{2}+q^{2}}}$ (where $\Delta$ is the pixel size). In other words, the trapezoidal shape involved in the filter regularization is itself adequately sampled. Eq.8 gives the result for the discrete bins $b$ onto the projection angle $(p, q)$. To avoid to sample the filtered projection at singularities locations (points where the trapezoidal shape gets two different derivatives), the Fourier implementation (Eq.6) can be preferred.

Secondly, the backprojection operator is implemented. This new scheme is compared with an implementation of the standard RamLaks FBP where the projection sampling is angle dependent or not (both cases were tested). Concerning this comparison, a very interesting point is the choice of the spatial location of the projection angles for the Mojette FBP case. As a matter of fact, the RamLaks FBP uses a regular angular sampling over $[0, \pi[$ whereas different choices are allowed for the Mojette FBP. This fact is due to the different nature of information that has been collected onto each bin: whereas a standard projector integrates the whole projection line, the Mojette Dirac projector only sums up the pixels values when the projection line intersects the center of the pixel.

For a given number of projections, different methods were implemented for the choice of the projections set giving the best reconstruction compared to the original image. Our test images are digital delta function and square phantom. To obtain $(p, q)$ angles satisfying Mojette geometry, we use Farey's series. ${ }^{4,12}$ The Farey series 
of order $N\left(F_{N}\right)$ is the set of all fractions in lowest terms between 0 and 1 whose denominators do not exceed $N$, arranged in order of magnitude. For example, $F_{4}$ is composed of $\left\{\frac{0}{1}, \frac{1}{4}, \frac{1}{3}, \frac{1}{2}, \frac{2}{3}, \frac{3}{4}, \frac{1}{1}\right\}$. Using symmetry, we found discrete angles into the whole space with the fraction denominator as $p$ and the numerator as $q$. For a square image $N \times N$, using the Farey's series of order $\mathrm{N}$, all the discrete directions to go from a pixel to an other one in the image are obtained. Our test images are $128 \times 128$, so we first generate $20088\left(C_{a r d}\left(F_{128}\right)\right)$ projections. Secondly, only 128 projections are selected using a tradeoff between a graceful degradation of the rigid angular sampling $\left(\frac{\pi}{128}\right)$ and a minimum number of bins. An angular sector is set around each continuous angle $\theta_{i}=\left\{\frac{i \pi}{128}, i \in[0 . .127]\right\}$ and the closest discrete angle in the Farey's series $F_{128}$ with the minimum number of bins is chosen in this angular sector, i.e. the lowest $(p, q)$ value.

\section{COMPARISON WITH CLASSICAL FBP - SQUARE PHANTOM RECONSTRUCTION}

The presented images were reconstructed from a digital square phantom of $17 \times 17$ pixels centered in the $128 \times 128$ image with a Dirichlet condition ${ }^{13}$ with the value 1 in the centered $15 \times 15$ square, $\frac{1}{2}$ in the boundaries and $\frac{1}{4}$ in the corners. In Fig.3 and Fig.4, a comparison is made without the filter operator to show the structure appearing after the projection-backprojection stage both for the regular angle and the Mojette sampling.

Fig.3.a represents the original square phantom, Fig.3.b the reconstruction with the set of regular angles, and Fig.3.c the difference between these two images showing the null space artifacts as well as the filter properties. For the usual projection-backprojection scheme each projection is composed of 128 bins. For the backprojection stage there is a linear interpolation onto the projections to get the bin values backprojected onto the pixel centers.

The reconstructed image is normalized. The maximum value is set to 1 , zero remains the same and other values are obtained by linear interpolation. The absolute difference is made between the original image and the normalized reconstructed image. The difference image is also normalized with the same method.

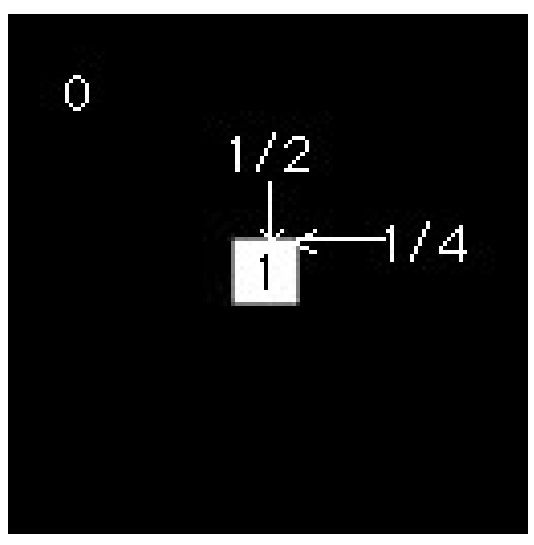

(a) Original image

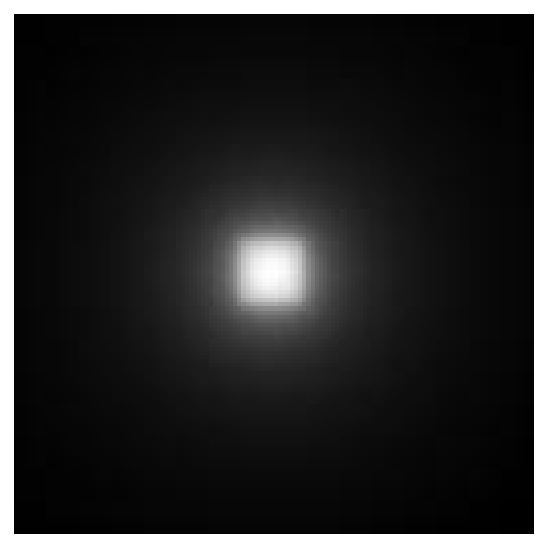

(b) Reconstructed image with classical backprojection

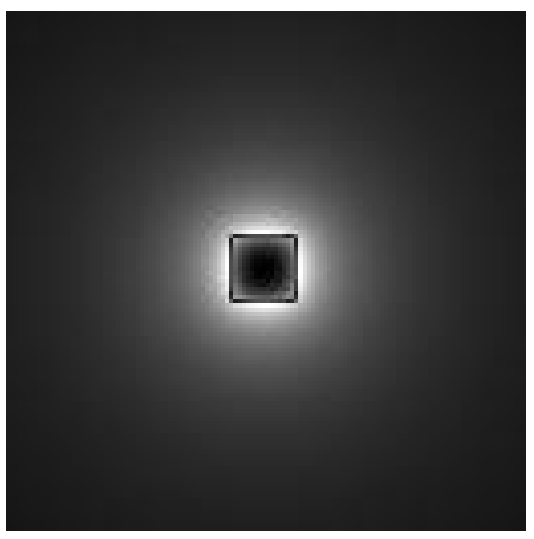

(c) Image error with a normalisation factor $\frac{1}{0.574}$

Figure 3. Image reconstruction with 128 gracefully distributed angles without filtering projections with a classical backprojection

Fig.4 shows the same images with the corresponding Mojette set of angles. Outside the square, according to the reconstruction method, the reconstruction is isotropic (see Fig.3.c) or anisotropic (see Fig.4.c). This depends on the angular sampling. The inside square phantom is better reconstructed with the Mojette backprojection according to the error images. This is particularly visible on the square corners and borders. The blur effect induced by the behavior of $M^{*} M$ (no filter implemented) is very different for the two methods. So its proves that the initial information (that sound very close) is also very different as well as the algorithms. 


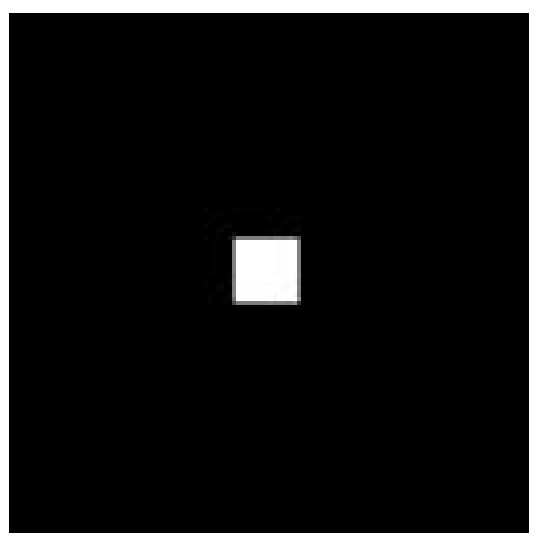

(a) Original image

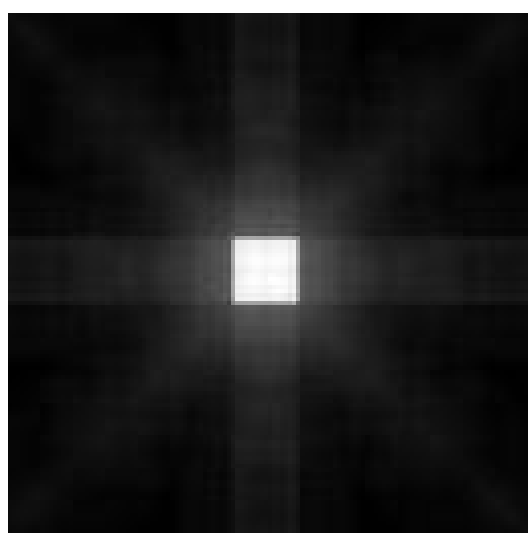

(b) Reconstructed image with a Mojette backprojection

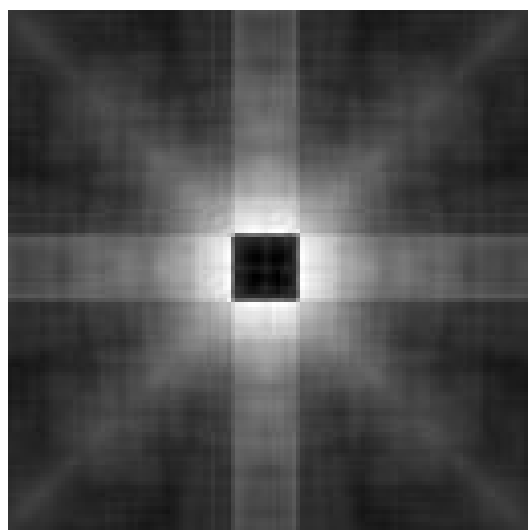

(c) Image error with a normalisation factor $\frac{1}{0.374}$

Figure 4. Image reconstruction with 128 gracefully distributed Mojette angles without filtering projections with a Mojette backprojection

Fig.5 and Fig.6 show the same results with an additional RAMLAKS filter. With the filtering stage, the anisotropic reconstruction outside the square disappears in the Mojette case. For this filter, the reconstruction gives better results inside the square with the classic FBP scheme except on the square corners perfectly reconstructed in the Mojette scheme.

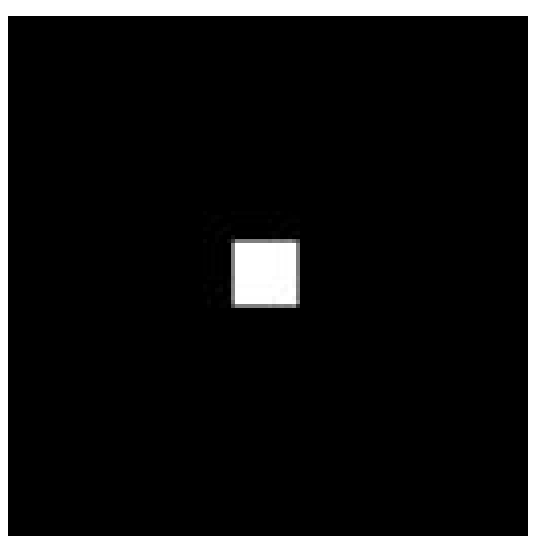

(a) Original image

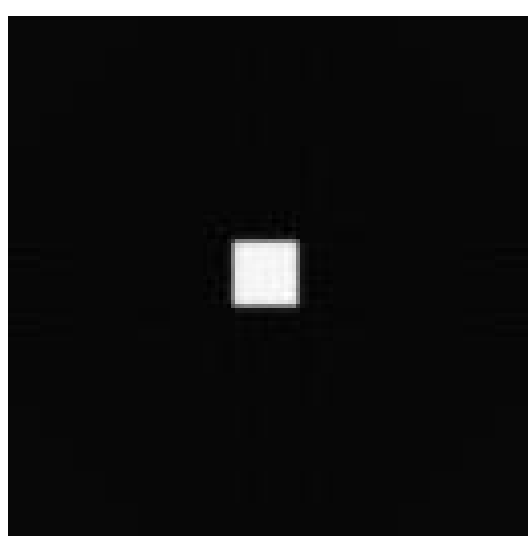

(b) Reconstructed image with a classical backprojection

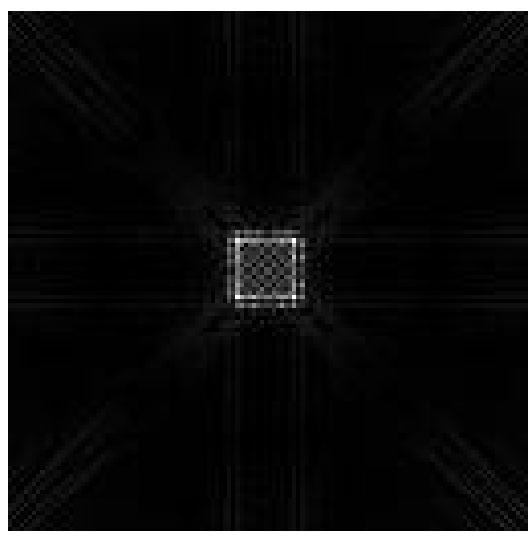

(c) Image error with a normalisation factor $\frac{1}{0.187}$

Figure 5. Image reconstruction with 128 gracefully distributed angles with convolution of projections with the RamLaks filter and a classical backprojection 


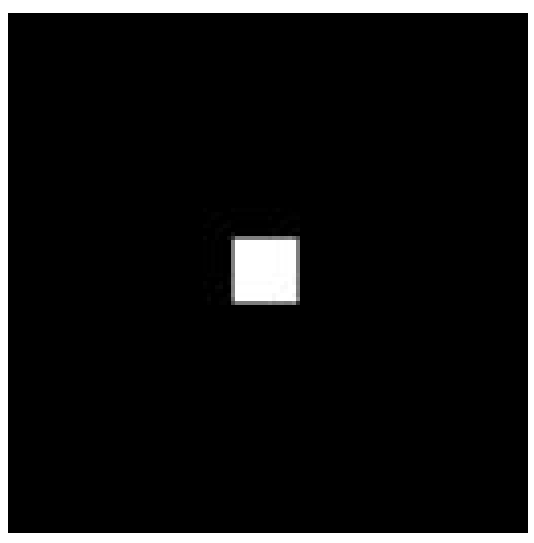

(a) Original image

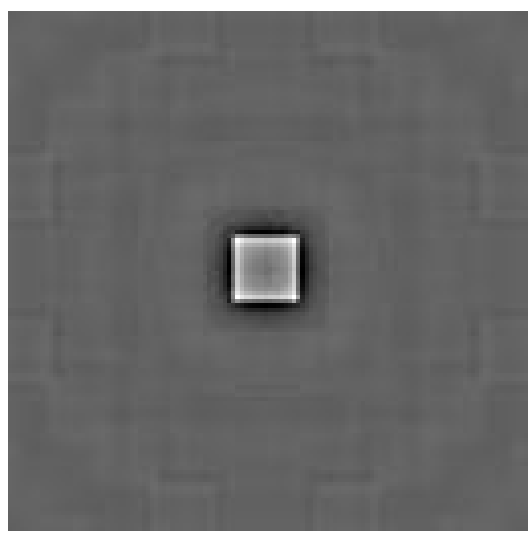

(b) Reconstructed image with a Mojette backprojection

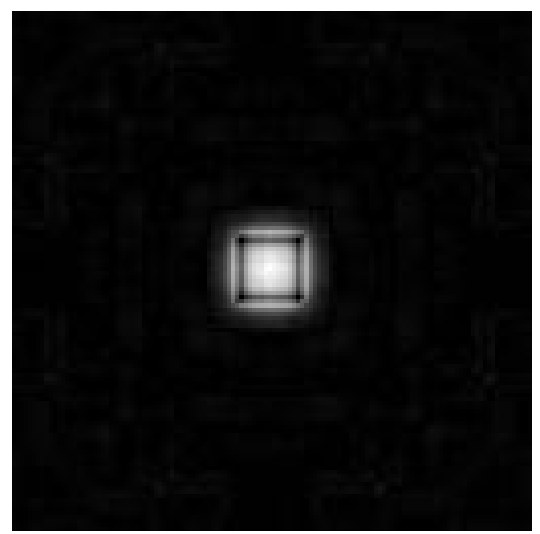

(c) Image error with a normalisation factor $\frac{1}{0.906}$

Figure 6. Image reconstruction with 128 gracefully distributed Mojette angles with convolution of projections with the RamLaks filter and a Mojette backprojection

Fig.7 and Fig.8 show the same results with the $k_{0}$ filter. The FBP scheme is performed with only 128 bins onto each projection. For the classic scheme computed with the $k_{0}$ filter, the square boundaries are not well reconstructed because the Dirichlet condition is not satisfied with no oversampling on the projections (see Fig.7.c). This result is in accordance with Guédon et al. ${ }^{1}$. The problem of no oversampling is the same in the Mojette case with the projections $(1,0)$ and $(0,1)$. There is only 128 bins onto these projections. All the others projections have more bins. The Dirichlet condition is not satisfied and there are some artifacts outside the square in these directions. Nevertheless the square is better reconstructed with the FBP Mojette scheme according to the error images.

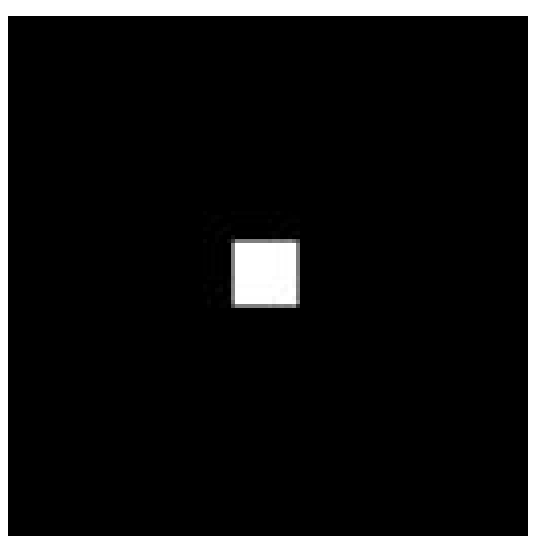

(a) Original image

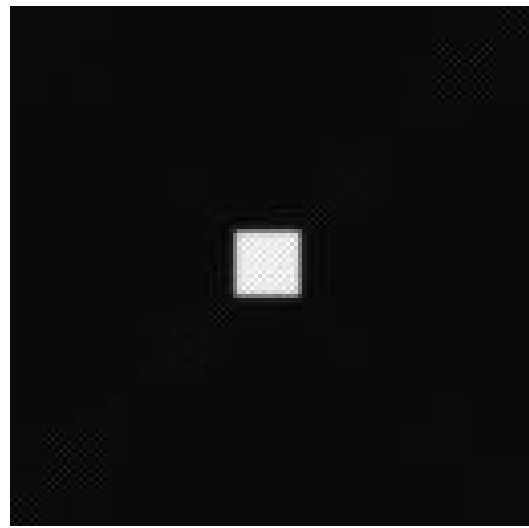

(b) Reconstructed image with a classical backprojection

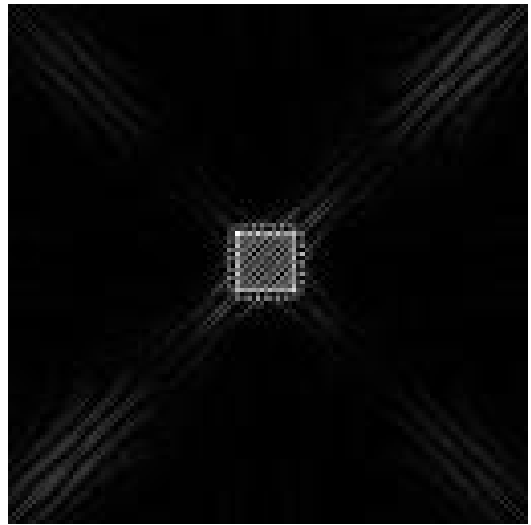

(c) Image error with a normalisation factor $\frac{1}{0.244}$

Figure 7. Image reconstruction with 128 gracefully distributed angles with convolution of projections with the $k_{0}$ filter and a classical backprojection 


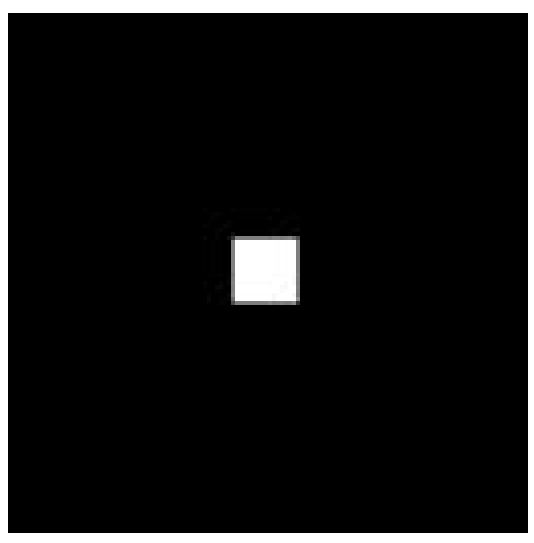

(a) Original image

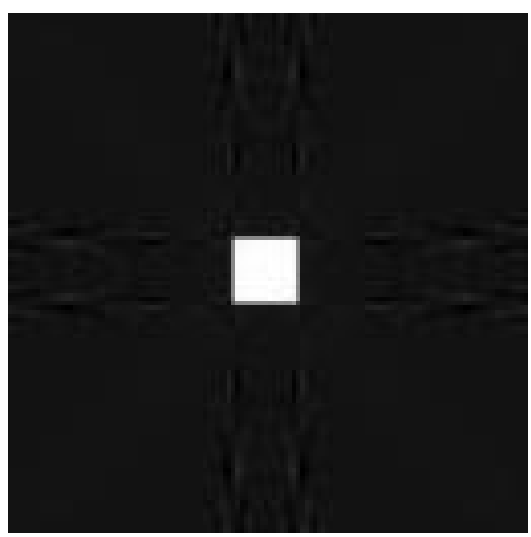

(b) Reconstructed image with a Mojette backprojection

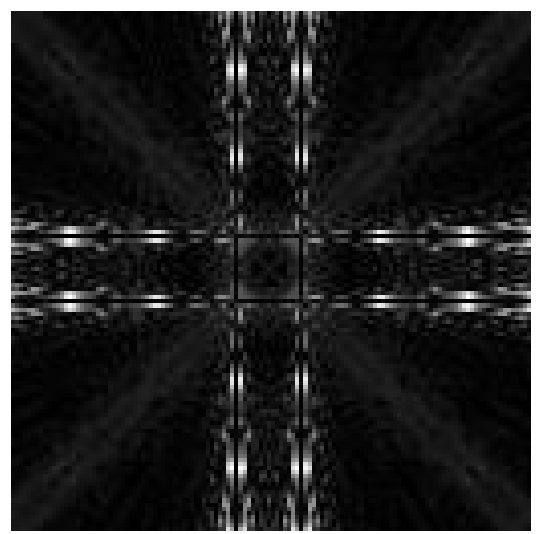

(c) Image error with a normalisation factor $\frac{1}{0.079}$

Figure 8. Image reconstruction with 128 gracefully distributed Mojette angles with convolution of projections with the $k_{0}$ filter with a Mojette backprojection

The error image on Fig.8.c corresponds to the error image with an oversampling of 4 onto the projections with a classic FBP scheme and a $k_{0}$ filter. ${ }^{1}$ The result is the same with oversampling onto classic projections or changing projections for geometric oversampled projections i.e. Mojette projections (except for the $(1,0)$ and the $(0,1)$ directions).

Finally, if we take a discrete set of angles close to the regular angular sampling, the best results are given by the FBP Mojette scheme using a $k_{0}$ filter.

\section{CHOICE OF THE DISCRETE ANGLES}

As previously explained (section 2.3.2), the 128 Mojette angles for this $128 \times 128$ test image are selected from a set of 20088 projections given by the Farey's series of order 128. These projections are gracefully distributed into $[0, \pi[$.

A second way of chosen the discrete angles was tested. Indeed, the Farey series of order 10 gives 128 projections angles (the couples $(p, q)$ associated to these angles are represented Fig. 9).

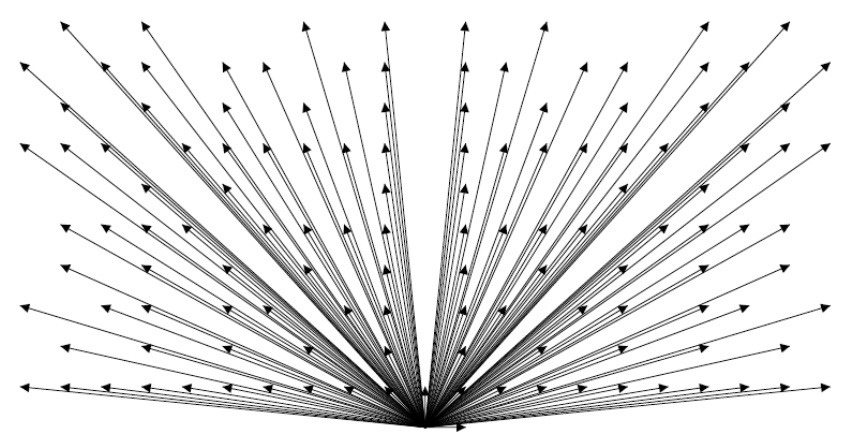

Figure 9. Discrete projection angles chosen with the Farey series of order 10 


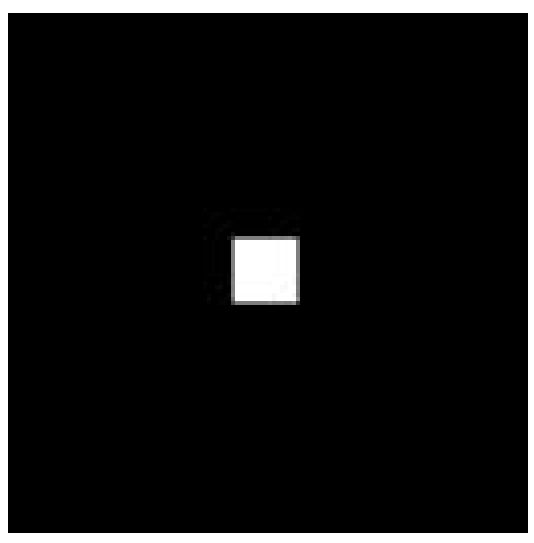

(a) Original image

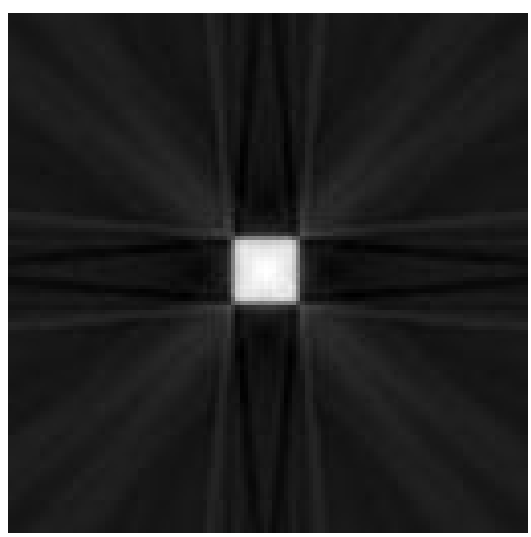

(b) Reconstructed image

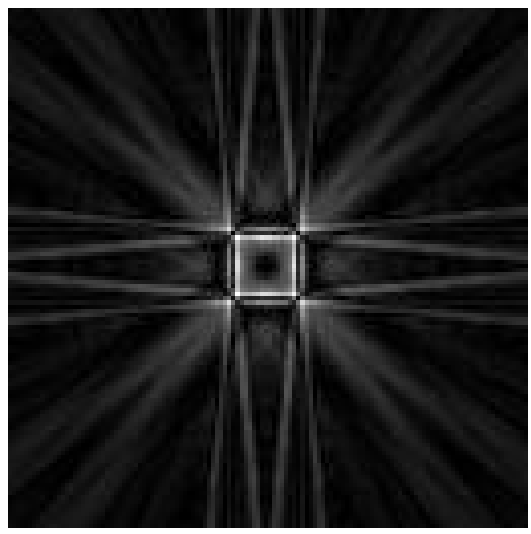

(c) Image error with a normalisation factor $\frac{1}{0.280}$

Figure 10. Image reconstruction with 128 angles (chosen with a Farey series of order 10) with convolution of projections with the $k_{0}$ filter with a Mojette backprojection

We can see that the 128 angles are not gracefully distributed and somes details as the square boundaries may not be well reconstructed or even do not appeared in the reconstructed image. Considering an angular sector in the horizontal, vertical and oblique directions there is some gaps in the space angular discretisation. In these directions the discrete angles are not well spaced. There is a lack of information in these directions which is confirmed by the error image Fig.10.c. A better result is obtained with a gracefully distributed set of discrete angles (Fig. 8) even if the angle gap problem remains around the horizontal, vertical and oblique directions.

\section{CONCLUSION}

In this paper the sampling aspects of the FBP scheme were revisited with the use of the Mojette transform. The reconstruction gives better results onto the square phantom for the FBP Mojette algorithm with the $k_{0}$ filter, especially on the corners and boundaries where the Dirichlet condition has to be satisfied. Using discrete angles implies both to get a nice angular and projection sampling. The angular sampling is better implemented from a selection of $I$ projections into a big Farey's series than restricting the order of magnitude of the Farey's series into $I$ generated projections. The projection sampling naturally given by the Mojette operator does not oversample the projections $0^{\circ}$ and $45^{\circ}$ but focuses onto the good compromise between projection bins and pixels with respect to the grid.

\section{REFERENCES}

1. J. Guédon and Y. Bizais, "Bandlimited and Haar Filtered Back-Projection Reconstuction," IEEE transaction on medical imaging 13, pp. 430-440, September 1994.

2. H. Barrett, "Fundamentals of the Radon transform." M.A. Viergever and A. Todd-Pokropek, eds., SpringerVerlag, Berlin, 1988.

3. A. Rosenfeld and A. Kak, Digital picture processing, Academic Press, Harcourt Brace Jovanovich, Publishers, 1982.

4. R. L. Graham, D. E. Knuth, and O. Patashnik, Concrete Mathematics: A Foundation for Computer Science (2nd Edition), Addison-Wesley, 1994.

5. N. Normand and J. Guédon, "La transformée Mojette : une représentation redondante pour l'image," Comptes-Rendus de l'Académie des Sciences de Paris, Informatique Théorique 325, pp. 123-126, 1997. 
6. A. Aldroubi and M. Unser, "Sampling procedures in function spaces and asymptotic equivalence with shannon's sampling theory," Numer. Funct. Anal. and Optimiz. 15, 1994.

7. J. Guédon, D. Barba, and N. Burger, "Psychovisual image coding via an exact discrete radon transform," SPIE Visual Communications and Image processing 2501, pp. 562-572, 1995.

8. M. Servières, N. Normand, P. Subirats, and J. Guédon, "Some links between continuous and discrete Radon Transform," in Medical Imaging 2004 : Image Processing, SPIE, 2004.

9. N. Normand and J. Guédon, "Spline Mojette transform. Application in tomography and communication," EUSIPCO II, pp. 407-410, 2002.

10. M. Servières, J. Guédon, and N. Normand, "A Discrete Tomography Approach to PET Reconstruction," in Fully 3D Reconstruction In Radiology and Nuclear Medicine, Y. Bizais, ed., June 2003.

11. M. Katz, Questions of Uniqueness and Resolution in Reconstruction from projections, vol. 26, SpringerVerlag, 1979.

12. I. Svalbe and A. Kingston, "Farey sequences and discrete Radon transform projection angles," in Electronic Notes in Discrete Mathematics, A. D. Lungo, V. D. Gesù, and A. Kuba, eds., 12, Elsevier, 2003.

13. J. Guédon, Sampling problems in tomography. Ph.D in Applied Computer Science (in French), University of Nantes, 1990. 